\title{
Mixoma odontogênico: estudo clínico-patológico de 14 casos
}

\section{Odontogenic myxoma: clinicopathological study of 14 cases}

\section{Cassiano Francisco Weege NONAKA}

Mestre - Programa de Pós-Graduação em Patologia Oral - Universidade Federal do Rio Grande do Norte UFRN - Natal - RN - Brasil

\section{Roberta Barroso CAVALCANTE}

Doutoranda - Programa de Pós-Graduação em Patologia Oral - Universidade Federal do Rio Grande do Norte UFRN - Natal - RN - Brasil

\section{Marta Rabelo PIVA}

Doutoranda - Programa de Pós-Graduação em Patologia Oral - Universidade Federal do Rio Grande do Norte UFRN - Natal - RN - Brasil

\section{Lélia Batista de SOUZA}

Professora Doutora - Programa de Pós-Graduação em Patologia Oral - Universidade Federal do Rio Grande do Norte UFRN - Natal - RN - Brasil

\section{Leão PEREIRA PINTO}

Professor Titular - Programa de Pós-Graduação em Patologia Oral - Universidade Federal do Rio Grande do Norte UFRN - Natal - RN - Brasil

\section{Resumo}

O propósito desta pesquisa consistiu em realizar estudo clínico-patológico de mixomas odontogênicos arquivados no Serviço de Anatomia Patológica do Departamento de Odontologia da Universidade Federal do Rio Grande do Norte. Ao todo, 14 casos de mixoma foram corados pela técnica de rotina da Hematoxilina e Eosina e examinados à microscopia de luz, descrevendo-se a presença de particularidades histopatológicas, como: áreas de intensa colagenização e remanescentes de epitélio odontogênico. Os dados clínicos referentes ao sexo e idade dos pacientes e localização anatômica das lesões foram obtidos em requisições de biópsias arquivadas. Evidenciou-se acometimento igualitário entre os sexos masculino e feminino. A média de idade dos pacientes ao diagnóstico foi de 33,07 anos, com o sexo masculino revelando valor médio superior (37,14 anos), em comparação com o feminino (29 anos). Em relação ao sítio anatômico, observouse predileção pela mandíbula $(57,14 \%)$, havendo maior acometimento da região posterior dos ossos gnáticos $(64,29$ $\%)$. Histologicamente, foram observadas áreas de intensa colagenização em meio ao estroma tumoral em $42,86 \%$ da amostra e 6 casos $(42,86 \%)$ revelavam ilhotas aparentemente inativas de epitélio odontogênico. Este estudo, apesar de concordar com os achados clínicos comumente relatados em análises retrospectivas de mixomas odontogênicos, descreve uma frequiência relativamente alta de áreas de intensa colagenização e remanescentes de epitélio odontogênico em meio à matriz extracelular destas neoplasias. Além disso, parâmetros para categorização do grau de colagenização da matriz extracelular de mixomas odontogênicos apresentam-se arbitrários e inconsistentes para classificação de mixomas e fibromixomas como entidades patológicas distintas.

\section{UNITERMOS}

Mixoma; tumor odontogênico. 


\section{INTRODUÇÃo}

Os mixomas são neoplasias benignas raras, representando cerca de 3 a $9 \%$ de todos os tumores odontogênicos ${ }^{10,13,18}$, e que se caracterizam histologicamente por intensa deposição de matriz gelatinosa, associada a quantidades variáveis de colágeno, assemelhando-se, estruturalmente, com o tecido da papila ou folículo dentário ${ }^{11}$.

A maioria dos casos é diagnosticada entre a segunda e a quarta décadas de vida, com pico de incidência na terceira década, sendo relatada uma discreta predileção por mulheres do que por homens, numa proporção de $2: 1^{10,13}$. Com relação ao sítio anatômico, a mandíbula encontra-se afetada em dois terços dos casos, em comparação com a maxila ${ }^{4,21}$.

Clinicamente, as lesões de pequeno volume são descobertas apenas durante exames radiográficos de rotina. Por sua vez, lesões maiores encontram-se associadas com expansão indolor do osso envolvido ${ }^{9,21}$. Radiograficamente, os mixomas odontogênicos apresentam-se como lesões radiolúcidas multiloculares, septadas por trabéculas ósseas retilíneas ou curvilíneas ${ }^{11}$.

Histologicamente, os mixomas revelam deposição de matriz mucóide ou gelatinosa, associada a quantidades variáveis de colágeno, com células exibindo morfologia fusiforme ou estrelada, com longos prolongamentos citoplasmáticos, usualmente, sem a presença de figuras de mitose ${ }^{3,5,23}$. A estes achados, Halfpenny et al. ${ }^{6}$ (2000) associam a presença de ocasionais ilhotas de epitélio odontogênico inativas.

Em certos casos, os mixomas podem apresentar maior tendência à formação de fibras colágenas, sendo denominados de fibromixomas ou mixofibromas ${ }^{7}$. Adicionalmente, achados incomuns são relatados, como a presença de ilhotas epiteliais revelando degeneração microcística ${ }^{8} \mathrm{e}$ áreas mineralizadas sob a forma de material cemento-like ou osteocemento-like ${ }^{14}$. Além disso, raramente, os mixomas podem apresentar acentuada celularidade e atipias celulares, com curso clínico mais agressivo, sendo, nestas situações, denominados de mixossarcomas ${ }^{15}$.

As modalidades terapêuticas variam desde curetagem à ressecção em bloco, com margens cirúrgicas amplas $^{21}$. As recorrências apresentam-se em torno de $25 \%$ dos casos, sugerindo-se a infiltração local como principal responsável por este índice ${ }^{23}$.

Em decorrência da freqüência relativamente baixa dos mixomas odontogênicos e, conseqüentemente, do reduzido número de estudos com maior casuística, constituiu-se objetivo desta pesquisa, realizar uma análise clínico-patológica dos casos arquivados no Serviço de Anatomia Patológica do Departamento de Odontologia da Universidade Federal do Rio Grande do Norte (UFRN).

\section{Material e Métodos}

\section{Amostra}

Um total de 14 espécimes de mixomas odontogênicos, diagnosticados no Serviço de Anatomia Patológica do Departamento de Odontologia da UFRN, foram utilizados nesta pesquisa. Os critérios determinados para inclusão de casos na amostra consistiram na localização das lesões e a quantidade de material biológico dos espécimes emblocados em parafina.

Em decorrência das discussões na literatura sobre as formas periféricas de mixomas, foram utilizados apenas casos de mixomas com localização intra-óssea, associados de forma mais consistente à histogênese odontogênica. Qualquer espécime que não obedeceu aos critérios descritos anteriormente foi descartado da pesquisa.

\section{Estudo Clínico e Morfológico}

Para o estudo clínico, foram utilizadas as informações constantes nas fichas de requisição de biópsias, arquivadas no Serviço referenciado anteriormente. Coletaram-se os dados referentes ao sexo e idade dos pacientes e localização anatômica das lesões. Para o estudo morfológico, utilizaram-se lâminas com cortes de $5 \mu \mathrm{m}$ de espessura do material emblocado em parafina, corados pela técnica de rotina da Hematoxilina/ Eosina e examinados à microscopia de luz, com aumentos de 100x e 400x (Microscópio Olympus CH30).

Os espécimes de mixomas odontogênicos foram analisados descritivamente, levando-se em consideração, a presença de particularidades histopatológicas arroladas de forma consistente na literatura, como nos trabalhos de Kumar, Jain e Gupta (2002), Frezzini et al. (2003) e Simon et al. (2004), determinando os valores percentuais dos seguintes parâmetros: áreas de intensa colagenização e remanescentes de epitélio odontogênico.

\section{Implicações Éticas}

O projeto de pesquisa ora desenvolvido foi submetido à apreciação do Comitê de Ética em Pesquisa da UFRN, sendo aprovado através do parecer $n^{\circ}$ $156 / 2005$. 


\section{Resultados}

Os dados clínicos dos casos de mixoma odontogênico (Tabela 1), obtidos nas fichas de biópsia dos pacientes submetidos a procedimento cirúrgico, demonstraram acometimento igualitário no tocante ao número de ocorrências entre os sexos masculino e feminino $(50 \%)$. A idade dos pacientes incluídos neste estudo variou de 07 a 60 anos, constatandose um valor médio ao diagnóstico da condição de 33,07 anos. Pôde-se evidenciar que os homens apresentaram maior média de idade ao diagnóstico da condição (37,14 anos), em comparação com as mulheres (29 anos).

Com relação ao sítio anatômico envolvido pelo tumor, os dados demonstraram predileção pela mandíbula $(57,14 \%)$, evidenciando-se menor ocorrência de casos na maxila $(35,71 \%)$. Por sua vez, avaliandose as regiões anterior e posterior dos ossos gnáticos, evidenciou-se que a primeira apresentou menor freqüência de casos $(21,43 \%)$ em comparação com a segunda $(64,29 \%)$.

Tabela 1 - Dados clínicos referentes ao sexo, idade, localização anatômica e região acometida, dos casos de mixomas odontogênicos

\begin{tabular}{c|c|c|cc}
\hline Caso & Sexo & Idade (em anos) & $\begin{array}{c}\text { Localização } \\
\text { Anatômica }\end{array}$ & Região \\
\hline 1 & Masculino & 32 & Maxila & Posterior \\
2 & Masculino & 28 & N/E & Posterior \\
3 & Feminino & 55 & Mandíbula & Posterior \\
4 & Feminino & 7 & Maxila & Anterior \\
5 & Feminino & 17 & Maxila & Posterior \\
6 & Feminino & 26 & Mandíbula & Posterior \\
7 & Masculino & 42 & Mandíbula & Anterior \\
8 & Feminino & 31 & Mandíbula & Posterior \\
10 & Feminino & 20 & Mandíbula & Posterior \\
11 & Masculino & 60 & Maxila & Posterior \\
13 & Masculino & 14 & Mandíbula & Anterior \\
14 & Masculino & 26 & Mandíbula & Posterior \\
\hline
\end{tabular}

Legenda: N/E - não especificado.

\section{Resultados Morfológicos}

A análise morfológica dos casos de mixoma odontogênico revelou como achado característico ao referido neoplasma, a presença de abundante tecido de aspecto mixóide, interposto por delgados feixes de fibras colágenas e poucos vasos sangüíneos de diâmetros variáveis. Além disso, verificaram-se escassos fibroblastos exibindo morfologia variando de estrelada a fusiforme, associados a prolongamentos citoplasmáticos delgados e alongados, exibindo, ocasionalmente, citoplasma intensamente eosinofílico e núcleos densamente basofílicos (Figura 1).
Dentre os parâmetros morfológicos avaliados, observou-se que 06 espécimes $(42,86 \%)$ exibiam áreas de intensa colagenização em meio ao estroma tumoral (Figura 2) e, 06 casos $(42,86 \%)$, revelavam ilhotas aparentemente inativas de epitélio odontogênico (Figura 3). Não obstante, características descritas com menor freqüência na literatura também foram constatadas, como a presença de fragmentos remanescentes de osso trabecular e ocasionais células inflamatórias, representadas por linfócitos e plasmócitos. Entretanto, pleomorfismo celular, nucléolos proeminentes e figuras de mitose não foram observados. 


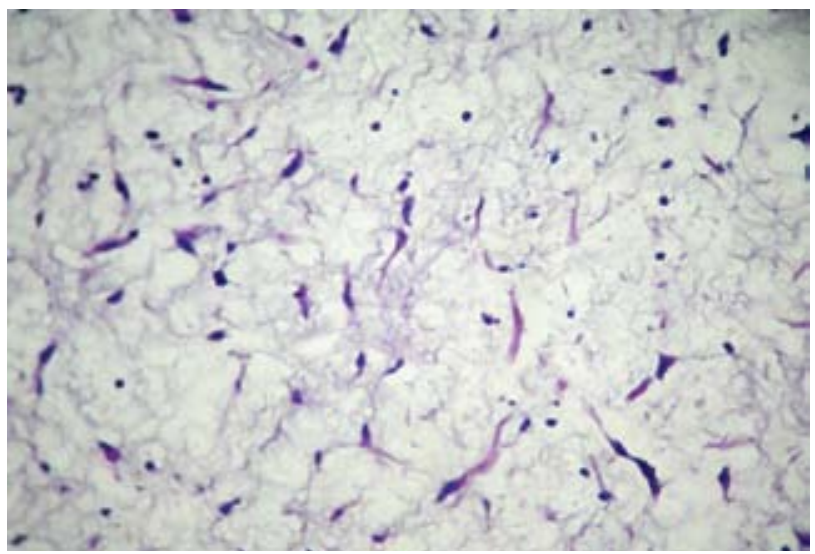

FIGURA 1 - Fotomicrografia revelando abundante tecido de aspecto mixóide, interposto por escassos fibroblastos exibindo morfologia variando de estrelada a fusiforme, associados a prolongamentos citoplasmáticos delgados e alongados $(\mathrm{H} / \mathrm{E}-400 \mathrm{x})$.

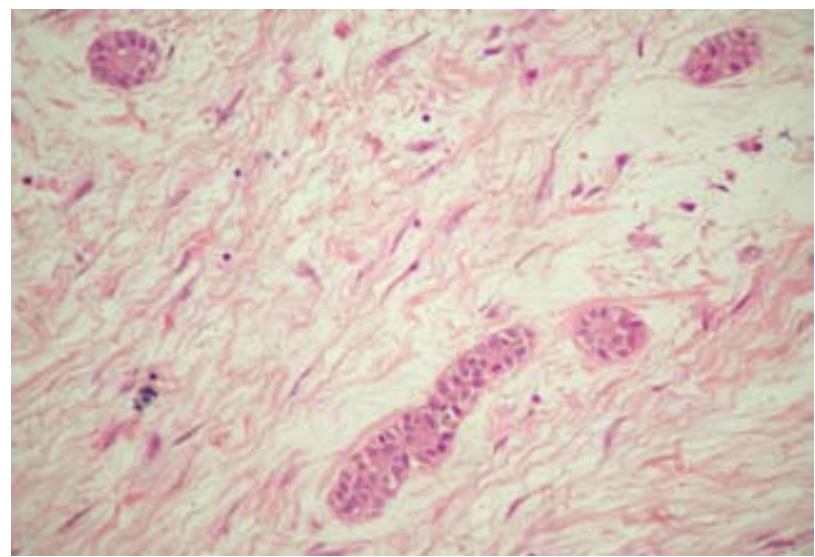

\section{Discussão}

Diversos estudos ${ }^{10,11,13,18,20}$ revelam resultados similares e consistentes em relação às características clínico-patológicas demonstradas pelos mixomas odontogênicos, apesar dos numerosos questionamentos acerca dos aspectos histogenéticos e patogenéticos desta neoplasia.

A despeito da ocasional variação na idade dos pacientes acometidos por mixomas odontogênicos ${ }^{5,14}$, numerosos estudos retrospectivos ${ }^{3,10,18,20,21,22}$, incluindo a pesquisa ora conduzida, revelam que a maior proporção dos casos é diagnosticada entre a segunda e a quarta décadas de vida.

Lo Muzio et al. ${ }^{12}$ (1996), analisando retrospectivamente 10 casos de mixomas odontogênicos,

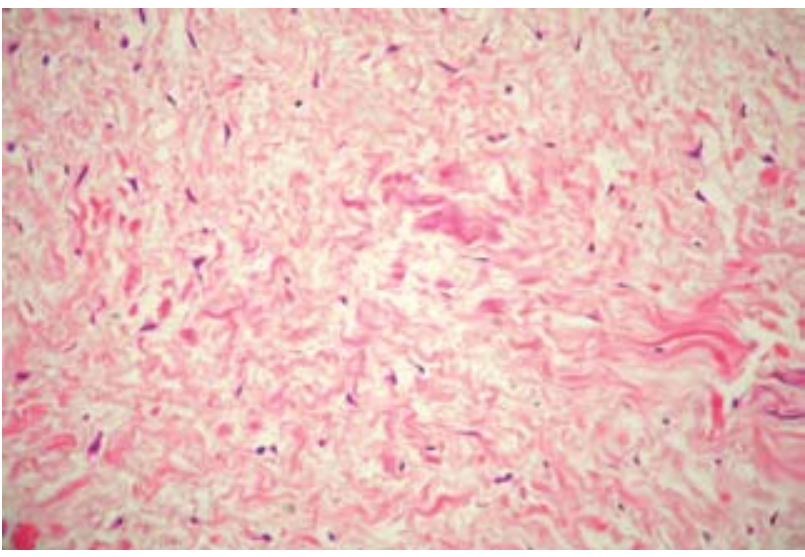

FIGURA 2 - Área de intensa colagenização, exibindo feixes espessos e alongados, em meio ao estroma tumoral de mixoma odontogênico $(\mathrm{H} / \mathrm{E}-400 \mathrm{x})$
FIGURA 3 - Ilhotas aparentemente inativas de epitélio odontogênico, dispersas em meio ao estroma tumoral de mixoma odontogênico $(\mathrm{H} / \mathrm{E}-400 \mathrm{x})$ evidenciaram que a distribuição destes em pacientes do sexo masculino ocorreu em idades entre 17 e 28 anos, tendo-se como média 22 anos, ao passo que no sexo feminino, as idades oscilaram entre 15 e 65 anos, com média de 38,8 anos. Tais resultados discordam do constatado em nossa pesquisa, na qual os homens apresentaram maior média de idade ao diagnóstico da condição (37,14 anos), em comparação com as mulheres (29 anos).

Diversos estudos epidemiológicos descrevem um maior acometimento do sexo feminino, nos casos de mixomas odontogênicos. Simon et al..$^{21}$ (2004) observaram que dos 33 casos diagnosticados em seu serviço, $21(63,64 \%)$ ocorreram em mulheres e apenas 12 
$(36,36 \%)$ acometeram indivíduos do sexo masculino. Valores percentuais semelhantes foram identificados por Ladeinde et al. ${ }^{10}$ (2005), os quais relataram 14 casos de mixoma (66,7\%) em mulheres e $07(33,3$ $\%)$ em indivíduos do sexo masculino.

Contudo, trabalhos com número amostral menor revelam valores percentuais mais próximos. Tamme et al. ${ }^{22}$ (2004) descreveram 09 casos de mixomas, sendo 05 destes $(55,56 \%)$ no sexo feminino e $04(44,44 \%)$ no masculino. Não obstante, Santos et al. ${ }^{18}$ (2001), em seu estudo epidemiológico sobre tumores odontogênicos, descreveram uma distribuição igualitária entre os sexos, com 03 casos (50\%) acometendo o sexo masculino e $03(50 \%)$ afetando o sexo feminino. Este último trabalho apresenta resultados concordantes com o verificado no estudo ora realizado, no qual evidenciou-se uma distribuição equivalente entre homens e mulheres, com 07 casos acometendo cada um destes.

Analisando o sítio de predileção dos mixomas odontogênicos, diversos trabalhos relatam maior acometimento da mandíbula em relação à maxila, em proporções que variam de $53 \%$ a $75 \%$ 1,3,10,13,21,22, faixa que contempla o valor percentual obtido nesta pesquisa, onde a mandíbula revelou-se acometida em $57,14 \%$ dos casos. Com relação às regiões dos ossos gnáticos, estudos epidemiológicos ${ }^{18,22} \mathrm{e}$ análises específicas em crianças e adolescentes ${ }^{2,7}$ afirmam que a porção posterior apresenta maiores taxas de acometimento, as quais variam entre $62,5 \%$ e 88,89 $\%$, intervalo que contém o valor evidenciado neste estudo $(64,29 \%)$.

Diversos estudos na literatura, sob a forma de relatos de $\operatorname{casos}^{5,11,16,19}$ e análises retrospectivas de $\operatorname{casos}^{7,12,17,21}$, descrevem características morfológicas, sejam estas relacionadas aos componentes estromais ou parenquimatosos, encontradas com maior ou menor frequiência nos mixomas odontogênicos.

Simon et al. ${ }^{21}$ (2004) afirmam que alguns casos de mixomas odontogênicos avaliados em seu estudo apresentavam quantidades moderadas de fibras colágenas, sem, entretanto, classificá-los como fibromixomas. Contudo, Keszler et al.7 (1995), apesar de não fornecerem parâmetros para quantificar a deposição de fibras colágenas observada nos 10 casos de mixomas odontogênicos avaliados em seu estudo, denominaram 03 destes como fibromixomas.

Lo Muzio et al. ${ }^{12}$ (1996) também descreveram 03 casos nos quais se evidenciavam numerosos feixes colagênicos espessos. Entretanto, estes autores não categorizaram seus casos como fibromixomas. Por sua vez, no estudo ora realizado, 06 casos $(42,86 \%)$ apresentavam áreas de intensa colagenização, representadas por feixes espessos de fibras colágenas, dispostos de forma localizada, entre abundante quantidade de substância fundamental de aspecto mixóide.

Freqüências elevadas, com relação à deposição colagênica, também foram evidenciadas no estudo de Rotenberg et al. ${ }^{17}$ (2004). Em seu trabalho, analisando 05 casos de mixomas odontogênicos localizados exclusivamente na maxila de crianças abaixo de 14 anos de idade, os autores classificaram 03 destes $(60$ $\%)$ como mixomas e $02(40 \%)$ como fibromixomas. Apesar de não pormenorizarem os parâmetros utilizados nesta categorização, os autores enfatizam que a quantidade de colágeno verificada nestes casos não se correlacionava com a idade da criança ou com o tempo de evolução do tumor.

Em virtude do reduzido número de estudos que avaliam a deposição colagênica na matriz extracelular dos mixomas odontogênicos, com intuito de categorizar mixomas e fibromixomas ${ }^{7,17}$, bem como, em decorrência da dificuldade de se estabelecerem parâmetros para determinar a quantidade de fibras colágenas presentes nestas lesões ${ }^{6,12,14,21}$, aliado, ainda, à ausência de pesquisas analisando diferenças no comportamento biológico em relação ao tipo de estroma predominante observado, o trabalho ora consubstanciado corrobora as assertivas de Keszler et al. ${ }^{7}$ (1995), não considerando mixomas e fibromixomas como entidades patológicas distintas.

Além disso, para Keszler et al. ${ }^{7}$ (1995), os mixomas odontogênicos demonstram predominância de células estreladas, dispostas em matriz de aspecto gelatinoso, com ocasionais fibras colágenas presentes na periferia dos espécimes, ao passo que os fibromixomas apresentam feixes colágenos e vasos sangüíneos dispostos de forma mais significativa. Apesar de constatar-se deposição colagênica de forma consistente em áreas dos espécimes utilizados no presente estudo, não se evidenciaram diferenças com relação ao padrão de vascularização, o qual apresentou-se constituído por escassos vasos sangüíneos, corroborando o descrito na literatura ${ }^{11,21}$.

Apesar de ser reportada a presença de remanescentes epiteliais odontogênicos, arranjados em pequenos ninhos em meio à substância fundamental de aspecto mixóide ${ }^{8}$, diversos trabalhos descrevem este achado como incomum à maioria dos mixomas ${ }^{14,17}$ e desnecessário ao diagnóstico da condição $0^{6,11}$.

Keszler et al. ${ }^{7}$ (1995), estudando 10 casos de mixomas odontogênicos, constataram remanescentes epite- 
liais em apenas 02 casos $(20 \%)$. Percentual idêntico foi constatado nos trabalhos realizados por Lo Muzio et al. ${ }^{12}$ (1996) e Rotenberg et al. (2004). No estudo ora realizado constatou-se uma freqüência relativamente alta de remanescentes epiteliais odontogênicos.

Dos 14 casos utilizados na amostra, $06(42,86 \%)$ revelaram ilhotas aparentemente inativas de epitélio odontogênico, as quais, por vezes, faziam-se numerosas, em meio à substância fundamental de aspecto mixóide. Deve-se ressaltar que a constatação de proporção elevada de remanescentes epiteliais, neste trabalho, em comparação com estudos de casuística semelhante, é plausível, em decorrência da existência de fragmentos da lâmina dentária, ao longo do interior dos ossos gnáticos.

Apesar da constatação dos remanescentes epiteliais odontogênicos na proporção citada anteriormente, em nenhum dos casos analisados neste estudo foram identificadas áreas de degeneração cística no interior das ilhotas, conforme descreveu o relato de caso incomum de Kimura et al. ${ }^{8}$ (2001).

Achados morfológicos descritos com menores freqüências também foram identificados nesta pesquisa, como a presença de trabéculas ósseas residuais ${ }^{19}$ e ocasionais células inflamatórias, representadas por linfócitos e plasmócitos ${ }^{11}$. Além disso, em nenhum dos casos analisados foram constatadas figuras de mitose, concordando com os resultados de Landa et al. ${ }^{11}$ (2002) e Simon et al. ${ }^{21}$ (2004) e divergindo do reportado por Fenton et al. ${ }^{4}$ (2003), os quais evidenciaram moderada atividade mitótica, com 04 mitoses por 10 campos de maior aumento (400x), em um caso de mixoma presente em uma criança de 1 ano e 5 meses de idade.

\section{Conclusão}

Este estudo, apesar de concordar com os achados clínicos comumente relatados em análises retrospectivas de mixomas odontogênicos, descreve uma frequiência relativamente alta de áreas de intensa colagenização e remanescentes de epitélio odontogênico em meio à matriz extracelular destas neoplasias.

Poucos estudos se propuseram a analisar aspectos morfológicos dos mixomas odontogênicos. Dentre os principais achados descritos na literatura, descrevemse áreas de intensa colagenização e a presença de remanescentes epiteliais odontogênicos. Parâmetros para categorização do grau de colagenização da matriz extracelular de mixomas odontogênicos apresentam-se arbitrários e, a princípio, são inconsistentes para classificação de mixomas e fibromixomas como entidades patológicas distintas.

\begin{abstract}
The purpose of this research was to perform a clinicopathological study in odontogenic myxomas presented on the files of Oral Pathology Service, Department of Dentistry, Federal University of Rio Grande do Norte. Fourteen cases of myxoma were stained with Hematoxylin and Eosin and examined under light microscopy, describing the presence of histopathologic features such as: areas of intense collagen deposition and remants of odontogenic epithelium. The clinical data related to the sex and age of patients and the tumor anatomical location were obtained from the dental records. The results revealed an equal distribution between male and female. The mean age of patients at the moment of diagnosis was 33,07 years, with male revealing a higher mean age (37,14 years) in comparison to female (29 years). Analyzing the anatomical location, it was revealed predilection for the mandible (57,14\%), with most of the cases located at the posterior region of gnathic bones (64,29\%). Histologically, it was observed areas of intense collagen deposition in the neoplasic stroma of 42,86\% of the sample and 6 cases (42,86\%) exhibitted islands of apparently inactive odontogenic epithelium. This research, despite of being concordant with the clinical data described in retrospective analysis of odontogenic myxomas, reveals a relativelly high frequency of areas of intense collagen deposition and remants of odontogenic epithelium lying in the extracellular matrix of such neoplasms. Nevertheless, parameters to establish the degree of colagenization of the extracellular matrix in odontogenic myxoma seem arbitrary and inconsistent to classify mixoma and fibromyxoma as distinct pathological entities.
\end{abstract}

\title{
UNITERMS
}

Myxoma; odontogenic tumour. 


\section{REFERÊNCIAS}

1. Adebayo ET, Ajike SO, Adekeye EO. A review of 318 odontogenic tumors in Kaduna, Nigeria. J Oral Maxillofac Surg. 2005; 63:811-9.

2. Adebayo ET, Ajike SO, Adekeye EO. Odontogenic tumours in children and adolescents: a study of 78 Nigerian cases. J CranioMaxillofac Surg. 2002; 30:267-72.

3. Dezotti MS, Azevedo LR, Fontão FG, Capelozza AL, Sant'ana E. Odontogenic myxoma - A case report and clinico-radiographic study of seven tumors. J Contemp Dent Pract. 2006; 7(1):117-24.

4. Fenton S, Slootweg PJ, Dunnebier EA, Mourits MP. Odontogenic myxoma in a 17-month-old child: a case report. J Oral Maxillofac Surg. 2003; 61:734-6.

5. Frezzini C, Maglione M, Rizzardini C, Melato M. Mixoma odontogeno recidivato dopo 11 anni: descrizione del caso clinico e considerazioni su una neoplasia rara. Minerva Stomatol. 2003; 52:247-51.

6. Halfpenny W, Verey A, Bardsley V. Myxoma of the mandibular condyle: a case report and review of the literature. Oral Surg Oral Med Oral Pathol Oral Radiol Endod. 2000; 90:348-53.

7. Keszler A, Dominguez FV, Giannunzio G. Myxoma in childhood: an analysis of 10 cases. J Oral Maxillofac Surg. 1995; 53:518-21.

8. Kimura A, Hasegawa H, Satou K, Kitamura Y. Odontogenic myxoma showing active epithelial islands with microscystic features. J Oral Maxillofac Surg. 2001; 59:1226-8.

9. Kumar N, Jain S, Gupta S. Maxillary odontogenic myxoma: a diagnostic pitfall on aspiration cytology. Diagn Cytopathol. 2002; 27(2):111-4.

10. Ladeinde AL, Ajayi OF, Ogunlewe MO, Adeyemo WL, Arotiba GT, Bamgbose BO et al. Odontogenic tumors: a review of 319 cases in a Nigerian teaching hospital. Oral Surg Oral Med Oral Pathol Oral Radiol Endod. 2005; 99:191-5.

11. Landa LE, Hedrick MH, Nepomuceno-Perez MC, Sotereanos GC. Recurrent myxoma of the zygoma: a case report. J Oral Maxillofac Surg. 2002; 60:704-8.

12. Lo Muzio L, Nocini P, Favia G, Procaccini M, Mignogna MD. Odontogenic myxoma of the jaws: a clinical, radiologic, immunohistochemical and ultrastructural study. Oral Surg Oral Med Oral Pathol. 1996; 82:426-33.

13. Ochsenius G, Ortega A, Godoy L, Peñafiel C, Escobar E. Odontogenic tumors in Chile: a study of 362 cases. J Oral Pathol Med. $2002 ; 31: 415-20$.

14. Oygür T, Dolanmaz D, Tokman B, Bayraktar S. Odontogenic myxoma containing osteocement-like spheroid bodies: report of a case with an unusual histopathological feature. J Oral Pathol Med. 2001; 30:504-6

15. Pahl S, Henn W, Binger T, Stein U, Remberger K. Malignant odontogenic myxoma of the maxilla: case with cytogenetic confirmation. J Laryngol Otol. 2000; 114:533-5.

16. Perez-Campos A, Perez-Rodriguez, F, Azorin D, Jimenez-Heffernan JA. Cytologic features of odontogenic myxoma. Acta Cytol. 2004, 48:767-8.

17. Rotenberg BW, Daniel SJ, Nish IA, Ngan BY, Forte V. Myxomatous lesions of the maxilla in children: a case series and review of management. Int J Pediatr Otorhinolaryngol. 2004; 68:1251-6.

18. Santos JN, Pereira Pinto L, Figueiredo CR, Souza LB. Odontogenic tumors: analysis of 127 cases. Pesqui Odontol Bras. 2001 15(4):308-13.

19. Shimoyama T, Horie N, Kato T, Tojo T, Nasu D, Kaneko T et al. Soft tissue myxoma of the gingiva: Report of a case and review of the literature of soft tissue myxoma in the oral region. J Oral Sci. 2000; 42(2):107-9.

20. Simon EN, Merkx MA, Vuhahula E, Ngassapa D, Stoelinga PJ. A 4-year prospective study on epidemiology and clinicopathological presentation of odontogenic tumors in Tanzania. Oral Surg Oral Med Oral Pathol Oral Radiol Endod. 2005; 99:598-602.

21. Simon EN, Merkx MA, Vuhahula E, Ngassapa D, Stoelinga PJ. Odontogenic myxoma: a clinicopathological study of 33 cases. Int J Oral Maxillofac Surg. 2004; 33:333-7.

22. Tamme T, Soots M, Kulla A, Karu K, Hanstein S, Sokk A et al. Odontogenic tumours, a collaborative retrospective study of 75 cases covering more than 25 years from Estonia. J CranioMaxillofac Surg. 2004; 32:161-5.

23. Triana MG, Monroy JL. Fibromixoma odontogénico. An Pediatr. 2005; 62(4):370-2

Recebido em: 21/12/06

Aprovado em: 20/04/07

Correspondência para: Prof. Dr. Leão Pereira Pinto e-mail: lppinto@digi.com.br / cassiano_nonaka@yahoo.com.br Programa de Pós-Graduação em Patologia Oral Departamento de Odontologia Universidade Federal do Rio Grande do Norte (UFRN)

Av. Senador Salgado Filho, 1787 - Lagoa Nova, Natal - RN Brasil - CEP: 59056-000 